\title{
Vitamin B12 Replacement Therapy After Gastrectomy
}

ISSN: 2637-7632

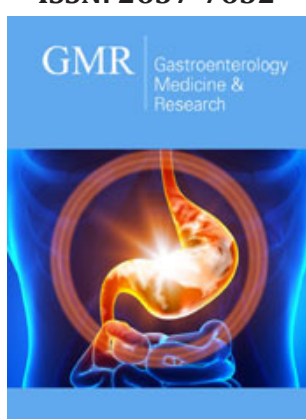

*Corresponding author: Yasushi Rino, Department of Surgery, Yokohama City University Graduate School of Medicine, 3-9, Fukuura, Kanazawa-ku, Yokohama, 236-0004, Japan

Submission: 傮 March 03, 2021

Published: 䟧 March 15, 2021

Volume 5 - Issue 4

How to cite this article: Yasushi Rino*, Toru Aoyama, Takanobu Yamada, Yosuke Atsumi, Hiroshi Tamagawa, Takashi Oshima, Tsutomu Sato, Norio Yukawa. Vitamin B12 Replacement Therapy After Gastrectomy. Gastro Med Res. 5(4). GMR. 000620. 2021.

DOI: 10.31031/GMR.2021.05.000620

Copyright@ Yasushi Rino, This article is distributed under the terms of the Creative Commons Attribution 4.0 International License, which permits unrestricted use and redistribution provided that the original author and source are credited.
Yasushi Rino ${ }^{*}$, Toru Aoyama ${ }^{1}$, Takanobu Yamada $^{2}$, Yosuke Atsumi ${ }^{1}$, Hiroshi Tamagawa $^{1}$, Takashi Oshima ${ }^{2}$, Tsutomu Sato ${ }^{3}$ and Norio Yukawa ${ }^{1}$

${ }^{1}$ Department of Surgery, Yokohama City University, School of Medicine, Yokohama, 3-9, Fukuura, Kanazawa-ku, Yokohama city, 236-0004, Japan

${ }^{2}$ Department of Gastrointestinal Surgery, Kanagawa Cancer Center, Yokohama, 2-3-2, Nakao, Asahi-ku, Yokohama city, 241-8515, Japan

${ }^{3}$ Gastroenterological Center, Medical Center, Yokohama City, Yokohama, 4-57 Urafune, Minami-ku, Yokohama 232-0024 Japan

\begin{abstract}
Vitamin B12 absorption has two mechanism that are passive and active one. The active mechanism, controlled gastric intrinsic factor, occurs through the ileum and is efficient for small oral dose of vitamin B12. After gastrectomy, there is little intrinsic factor. Especially, after total gastrectomy (TG), intrinsic factor is lost. Cumulative vitamin B12 deficiency rate was $100 \%$ for TG after surgery. Usually, intramuscular injection of vitamin B12 for vitamin B12 deficiency after gastrectomy is performed. Recently, oral vitamin B12 replacement was reported. $500 \mu \mathrm{g}$ vitamin B12 replacement orally is maybe effective and necessary.
\end{abstract}

Keywords: Vitamin B12; Replacement therapy; Total gastrectomy; Gastric cancer; Oral vitamin B12 replacement

Abbreviations: TG: Total Gastrectomy; DG: Distal Gastrectomy

\section{Introduction}

\section{Mechanisms of vitamin B12 absorption}

Mechanisms of vitamin B12 absorption has been known widely. The Mechanisms are classified into two mechanisms. One is passive, occurring equally through buccal, duodenal, and ileal mucosa; it is rapid but extremely inefficient, with $<1 \%$ of an oral dose being absorbed by this process. The other one is active; it occurs through the ileum and is efficient for small oral dose of vitamin B12, and it is mediated be substance named gastric intrinsic factor. Intrinsic factor is produced in the gastric parietal cells of the fundus and body of the stomach. Between 0.5-5 $\mu$ g of cobalamin excreted with the bile each day. This binds to intrinsic factor, and a major portion of biliary cobalamin normally is reabsorbed together with cobalamin derived from sloughed intestinal cells. Because of the loss of these effective absorption and reabsorption mechanism, vitamin B12 deficiency develops more rapidly after gastrectomy $[1,2]$.

\section{Loss of vitamin B12 absorption mechanisms after gastrectomy}

A variety of factors affects some patients after gastrectomy for gastric cancer. Some nutrition deficiencies result from disturbance of the normal anatomic and physiologic mechanism that control gastric function. After gastrectomy, patients commonly lose weight rapidly. The cause of weight loss, fat-soluble vitamin deficiency, and bone disorders after gastric surgery generally are decreased dietary intake or malabsorption [3-5]. Active mechanism of vitamin B12 absorption is almost lost after gastrectomy. Especially, after total gastrectomy (TG), patients have only passive mechanism of vitamin B12 absorption. Because of it, vitamin B12 deficiency after gastrectomy is common complication as malnutrition $[6,7]$. 


\section{Frequency of vitamin B12 deficiency after gastrectomy}

As above, cumulative vitamin B12 deficiency rates were 100\% for TG and $15.7 \%$ for distal gastrectomy (DG) 4 years after surgery. The median time to vitamin B12 deficiency was 15 months after TG, whereas the median time was not reached after DG [8]. We reported that about 9 months was the median time to vitamin B12 deficiency after TG [9]. Normally, between 2-3mg vitamin B12 are stored in human body. Because of this, vitamin B12 deficiency would not be occured. May TG progress a daily choler excretion or a consumption of the vitamin B12? Most gastric cancer patients are complicated with atrophic gastritis. A severe lack of intrinsic factor due to gastric atrophy [1]. Vitamin B12 storage before TG would be decreased in gastric cancer patients with gastric atrophy. If this hypothesis is correct, it can be inferred that the median time to vitamin B12 deficiency after TG is shortened.

\section{Treatment of vitamin B12 deficiency after gastrectomy}

In spite of vitamin B12 administration orally is not a reliable route after TG, Kim et al. [10] previously reported that serum vitamin B12 increased after oral and intramuscular administration of vitamin B12 in TG patients. In this report, for the oral vitamin B12 replacement, mecobalamin was administrated. The dosage comprised $1500 \mu$ g mecobalamin daily [10]. But we reported that $500 \mu \mathrm{g}$ vitamin B12 replacement orally is maybe effective [9].

\section{In the future}

Even after TG, patients have passive mechanism and can absorb little of oral intake vitamin B12. Vitamin B12 replacement therapy should be necessary and continued routinely. IM is a reliable therapy. But IM needs to go to clinic a month and is painful. According to our results, one tablet PO a day is enough [9]. And now, we planned clinical study to clarify $500 \mu \mathrm{g}$ vitamin B12 replacement orally for TG patients.

\section{Conflict of Interest Statement}

The authors declare no conflicts of interest in association with the present study.

\section{Acknowledgment}

None

\section{References}

1. Kasper DL, Fauci AS, Hauser SL, Longo DL, Jameson JL, et al. (2015) Harrison's principles of internal medicine. In: Hoffbrand AV (19 $\left.9^{\text {th }} \mathrm{Ed}\right)$, Megaloblastic anemias. McGraw-Hill, New York, USA, pp.640-649.

2. Brunicardi CF, Andersen DK, Billiar TR, Dunn DL, Hunter JG, et al. (2005) Schwartz's principles of surgery. In: Whang EE, Ashley SW, Zinner MJ ( $8^{\text {th }}$ Eds.), Small intestine. McGraw-Hill, New York, USA, pp.1017-1054

3. Aoyama T (2020) Perioperative body composition changes in the multimodal treatment of gastrointestinal cancer. Surg Today 50(3): 217222.

4. Rino Y, Oshima T, Yoshikawa $\mathrm{T}$ (2017) Changes in fat-soluble vitamin levels after gastrectomy for gastric cancer. Surg Today 47(2): 145-50.

5. Rino Y, Aoyama T, Atsumi Y, Yamada T, Yukawa N (2021) Metabolic bone disorders after gastrectomy: Inevitable or preventable?. Surgery Today.

6. Brunicardi CF, Andersen DK, Billiar TR, Dunn DL, Hunter JG, et al. (2005) Schwartz's principles of surgery. In: Dempsy DT (Ed.), Stomach. $\left(8^{\text {th }}\right.$ edn), McGraw-Hill, New York, USA, pp. 933-995.

7. Townsent CM, Beauchamp RD, Evers BM, Mattox KL (2012) Sabiston textbook of surgery. In: Mahvi DM, Krantz SK (19 ${ }^{\text {th }}$ Eds.), Stomach. Elsevier Saunders, Philadelphia, USA, pp. 1182-1226.

8. Hu Y, Kim HI, Hyung WJ, Song KJ, Lee JH, et al. (2013) Vitamin B (12) deficiency after gastrectomy for gastric cancer: An analysis of clinical patterns and risk factors. Ann Surg 258(6): 970-975.

9. Rino Y, Yamada T, Aoyama T, Numata M, Cho H, et al. (2018) Vitamin B12 replacement therapy after gastrectomy. J Clin Res Med 1(4): 1-4.

10. Kim HI, Hyung WJ, Song KJ, Choi SH, Kim CB, et al. (2011) Oral vitamin B12 replacement: An effective treatment for vitamin B12 deficiency after total gastrectomy in gastric cancer patients. Ann Surg Oncol 18(13): 3711-3717.

For possible submissions Click below: 